\title{
MICORRIZA ARBUSCULAR E MATÉRIA ORGÂNICA NA ACLIMATIZAÇÃO DE MUDAS DE BANANEIRA, CULTIVAR NANICÃO ${ }^{(1)}$
}

\author{
ROSA MARIA BARBOSA MATOS ${ }^{(2)}$; ELIANE MARIA RIBEIRO DA SILVA ${ }^{(3)}$; \\ FELIPE DA COSTA BRASIL ${ }^{(4,5)}$
}

\begin{abstract}
RESUMO
Com o objetivo de avaliar o efeito da inoculação de fungo micorrízico arbuscular na produção de mudas de qualidade, em sistema de pouco insumo, realizou-se um experimento com mudas micropropagadas de bananeira, cultivar Nanicão, em casa de vegetação do Centro Nacional de Pesquisa de Agrobiologia (Seropédica/RJ), em julho de 1998. O delineamento experimental empregado foi o de blocos ao acaso com quatro repetições, constituindo-se os tratamentos por três substratos (0,10\% e $20 \%$ de matéria orgânica), na presença e ausência de Glomus clarum. A partir dos 65 dias de aclimatização, as mudas desenvolvidas no substrato sem matéria orgânica, submetidas a inoculação com G. clarum, apresentaram efeito positivo significativo da inoculação na altura e no número de folhas. Aos 93 dias, as mudas infectadas, cultivadas no substrato com 0 e $10 \%$ de matéria orgânica, mostraram altura e diâmetro superior às não-infectadas. Na colheita, aos 95 dias, constatou-se aumento significativo da massa de folha, pseudocaule e raízes secas, bem como do conteúdo total de fósforo $(\mathrm{P})$ das mudas infectadas em relação às não-infectadas com $\mathrm{G}$. clarum. A avaliação da razão de eficiência radicular mostrou que as mudas dos substratos sem matéria orgânica e com $10 \%$, infectadas com G. clarum, apresentaram maior razão do que as não-infectadas. De maneira geral, a presença de matéria orgânica no substrato proporcionou efeito positivo no desenvolvimento das mudas de bananeira 'Nanicão'.

Palavras-chave: fungo micorrízico arbuscular, micropropagação, Glomus clarum, Musa spp.
\end{abstract}

\section{ABSTRACT \\ ARBUSCULAR MYCORRHIZAL AND ORGANIC MATTER ON THE ACCLIMATIZATION OF BANANA-TREE SEEDLINGS, CV. NANICÃO}

With the objective of evaluating the inoculation effect of arbuscular mycorrhizal fungus on the development of good-quality banana seedlings, cv. Nanicão, under a low-input system, a greenhouse experiment was done at Seropédica, State of Rio de Janeiro, Brazil, in the National Center of Agrobiology Research, in 1998. The experimental design was a randomized block, with four repetitions. The treatments consisted of growing micropropagated seedlings in three substrata with 0,10 and $20 \%$ of organic matter, in the presence or absence of the fungus Glomus clarum. The results showed a significant positive effect of the inoculation on seedling height and number of leaves, 65 days after acclimatization. At 93 days, this positive effect was shown also by the pseudostem diameter. After harvest, the seedlings inoculated with G. clarum showed a significant increase in leaf, pseudostem and root dry matter, as well as total phosphorus content and radicular ratio efficiency in comparison to non-inoculated seedlings. As a rule, the addition of organic matter to the substratum led to an increase in the development of banana seedlings, cv. Nanicão, even in the absence of G. clarum.

Key words: arbuscular mycorrhizal fungi, micropropagation, Glomus clarum, Musa spp.

(1) Extraído da tese de doutorado da primeira autora,apresentada à Universidade Federal Rural do Rio de Janeiro. Recebido para publicação em 11 de janeiro de 2002 e aceito em 17 de setembro de 2002.

$\left(^{2}\right)$ Agronomia - Área Ciência do Solo. E-mail:matosrmb@uol.com.br

${ }^{(3)}$ Embrapa Agrobiologia. Estrada Rio-São Paulo, km 47, Caixa Postal 74505, 23851-970 Seropédica (RJ). E-mail: eliane@cnpab.embrapa.br

$\left(^{4}\right)$ Doutorando em Agronomia, Área Ciência do Solo, Universidade Federal Rural do Rio de Janeiro. E-mail: febrasil@bol.com.br

$\left({ }^{5}\right)$ Com bolsa de produtividade científica do CNPq. 


\section{INTRODUÇÃO}

O Brasil é o maior produtor de banana da América do Sul, contribuindo com $37 \%$ na produção do continente (FAO, 1997). De modo geral, os cultivos seguem os padrões tradicionais, com baixos índices de capitalização e tecnologia, na maioria dos Estados brasileiros. Nos Estados de São Paulo, Santa Catarina e nos perímetros irrigados do Nordeste tem-se observado o uso de diferentes tecnologias que propiciam produção maior e de melhor qualidade (SOUZA e TORRES-FILHO, 1997).

Nos últimos anos, a propagação da bananeira por meio da cultura de ápices caulinares in vitro tem possibilitado a produção de mudas sadias, em número mais elevado que os métodos convencionais (SouzA et al., 1994). O desenvolvimento desta técnica para micropropagação do gênero Musa tem recebido considerável atenção nos últimos quinze anos e já se encontra bem estabelecida (CRONAUER e KRIKORIAN, 1984).

No emprego de mudas micropropagadas existe a necessidade de um período de aclimatização, em viveiro, para que as mudas adquiram condições de serem levadas ao campo. Nessa etapa, que se processa em situações controladas, utiliza-se substrato desinfestado, sendo de grande relevância a introdução de fungo micorrízico arbuscular (FMA). Os efeitos nutricionais são os mais evidentes e consistentes daqueles atribuídos às micorrizas, estando diretamente relacionados ao crescimento e à produção de plantas. Esse fato se deve, entre outros, a uma geometria mais favorável das hifas em relação às raízes da planta, à cinética de absorção de água e nutrientes diferentes e a alterações químicas na área de rizosfera/hifosfera que possibilitam maior e melhor distribuição da área de absorção (BoLAN, 1991). Deve-se considerar, contudo, que as espécies e cultivares podem diferir em seus requerimentos nutricionais devido, basicamente, a diferenças nas taxas de crescimento e na habilidade de captar e utilizar o fósforo (P) do solo (BARRow, 1977).

Pesquisas com diferentes culturas têm mostrado a importância do emprego de FMAs na produção de mudas micropropagadas (NIEMI e VESTBERG, 1992; LovATo et al., 1994). Na otimização deste processo considera-se, como fator de grande relevância, a composição do substrato. Diferentes estudos (SouzA et al., 1987; Verma E ARYA, 1998) demonstram a importância do esterco na composição de substratos para a produção de mudas. SouzA et al. (1989) verificaram, em mudas de café, que a presença de esterco de curral no substrato proporcionou a obtenção de maiores respostas para todas as características de crescimento avaliadas, não alterando a colonização das raízes por FMA.

Neste trabalho, objetivou-se avaliar os efeitos do FMA na aclimatização de bananeiras micropropagadas, em substratos com três níveis de matéria orgânica, visando à produção de mudas de qualidade em sistemas de baixo insumo.

\section{MATERIAL E MÉTODOS}

A partir de mudas micropropagadas de bananeira, cv. Nanicão, instalou-se um experimento em casa de vegetação da Embrapa Agrobiologia, no município de Seropédica (RJ), em julho de 1998, com duração de três meses. O delineamento experimental adotado foi o de blocos ao acaso com quatro repetições e quatro plantas por parcela, constituindose os tratamentos por três níveis de matéria orgânica $(0,10 \%$, e $20 \%$ de esterco de gado curtido), na presença e ausência do FMA Glomus clarum.

O substrato básico constituiu-se de uma mistura de solo Argissolo Vermelho-Amarelo e areia de rio lavada, na proporção de 2:1 (v:v), denominando-se esta mistura substrato 1 . Os substratos 2 e 3 foram constituídos pelo substrato 1 acrescido, respectivamente, de $10 \%$ e $20 \%$ de matéria orgânica, composta de esterco de gado curtido. Procedeu-se, em seguida, as determinações químicas dos substratos e da matéria orgânica (EMBRAPA, 1979) e a análise granulométrica dos substratos (EMBRAPA, 1997; Ravelli-Neto et al., 1994) - Quadros 1 e 2 . Com base na análise química realizou-se a adubação do substrato com $0 \%$ de matéria orgânica, utilizando-se superfosfato simples (50 mg. $\left.\mathrm{kg}^{-1}\right)$ e cloreto de potássio (25 mg. $\mathrm{kg}^{-1}$ ). Para a neutralização do Al usou-se calcário dolomítico $\left(500 \mathrm{mg} \cdot \mathrm{kg}^{-1}\right)$. Após a homogeneização do solo com os fertilizantes, realizaram-se as determinações químicas do substrato; os dados são apresentados no quadro 1 . Os substratos foram desinfestados com brometo de metila, de acordo com recomendações do fabricante, empregando-se como recipientes baldes de plástico branco, com capacidade de 10 litros.

No tratamento com FMA empregaram-se 140 esporos de G. clarum por planta, sendo este inóculo proveniente da coleção de cultura da Embrapa Agrobiologia. Para a reposição de microrganismos e da flora, eliminados pela desinfestação do substrato, preparou-se um filtrado com base em $2 \mathrm{~kg}$ de solo não brometado, ao qual se acrescentou $2,5 \mathrm{~L}$ de água. Essa mistura foi passada em peneira de $0,053 \mu \mathrm{m}$ e deixada em repouso para decantação. Após a finalização desse processo, realizaram-se duas 
Quadro 1. Análise química dos substratos com 0, 10 e 20 \% de matéria orgânica (estrume de curral curtido)

\begin{tabular}{|c|c|c|c|c|c|c|}
\hline Substrato & $\mathrm{pH}\left(\mathrm{H}_{2} \mathrm{O}\right)$ & $\mathrm{Al}$ & $\mathrm{Ca}$ & $\mathrm{Mg}$ & $\mathrm{P}$ & K \\
\hline & & \multicolumn{3}{|c|}{$\mathrm{mmol} \cdot \mathrm{dm}^{-3}$} & \multicolumn{2}{|c|}{$-\mathrm{mg} \cdot \mathrm{kg}^{-1}$} \\
\hline $0 \%$ & 5,4 & 0,0 & 20 & 9 & 7 & 63 \\
\hline $10 \%$ & 5,7 & 0,0 & 22 & 18 & 24 & 450 \\
\hline $20 \%$ & 6,5 & 0,0 & 28 & 18 & 37 & 704 \\
\hline Matéria orgânica & 8,4 & 0,0 & 48 & 62 & 375 & 5049 \\
\hline
\end{tabular}

Quadro 2. Análise granulométrica dos substratos com $0 \%, 10 \%$ e $20 \%$ de matéria orgânica (estrume de curral curtido)

\begin{tabular}{lcccccc}
\hline Substrato & $\begin{array}{c}\text { Areia } \\
\text { grossa }\end{array}$ & $\begin{array}{c}\text { Areia } \\
\text { fina }\end{array}$ & $\begin{array}{c}\text { Argila } \\
\text { total }\end{array}$ & $\begin{array}{c}\text { Grau de } \\
\text { floculação }\end{array}$ & $\begin{array}{c}\text { Densidade } \\
\text { real }\end{array}$ & $\begin{array}{c}\text { Condutividade } \\
\text { elétrica }\end{array}$ \\
\cline { 2 - 4 } $0 \%$ & 65 & 9 & 22 & & 9 & $\mathrm{mS.cm}^{-1}$ \\
$10 \%$ & 65 & 10 & 22 & 13 & 2,51 & 0,2 \\
$20 \%$ & 63 & 10 & 23 & 17 & 2,49 & 1,2 \\
\hline
\end{tabular}

filtragens em papel-filtro quantitativo (filtragem rápida - $50 \mathrm{~s}$ ). O filtrado resultante foi elevado a $2 \mathrm{~L}$, aplicando-se, em todos os vasos, uma razão de $20 \mathrm{~mL}$.

Avaliaram-se as mudas aos 32, 65 e 93 dias após o plantio, verificando-se a altura da planta, o diâmetro do pseudocaule à altura do colo e o número de folhas ativas. Na colheita, aos 95 dias de aclimatização, as plantas amostradas foram separadas em folhas, pseudocaule e sistema radicular. As folhas e pseudocaule foram colocadas em estufa a $65^{\circ} \mathrm{C}$, até a obtenção de massa seca constante, quando se realizou a pesagem. As raízes foram lavadas e separadas do substrato, sendo seus eixos recuperados em peneiras de $2 \mathrm{~mm}$. Os eixos radiculares foram digitalizados em scanner de mesa, com uma resolução de 100 dpi e quantização de 256 tons de cinza. As imagens obtidas foram analisadas com o auxílio do software SIARCS 3.0 (JORGE, 1996) sendo quantificadas a área superficial radicular e o comprimento radicular total. A seguir, separou-se 0,4 $\mathrm{g}$ de raízes finas para determinar a porcentagem de colonização micorrízica, sendo usado, para o clareamento e a coloração de raízes, o método de Koske e Gemma (1989). As raízes restantes foram colocadas na estufa para secagem, pesando-as consecutivamente até a obtenção de uma massa constante. A partir destas quantificações calcularamse os valores de área e comprimento radicular específico $\left(\mathrm{cm}^{2} \cdot \mathrm{g}^{-1} \mathrm{e} \mathrm{mg}^{-1}\right)$.

$\mathrm{Na}$ parte aérea e nas raízes secas em estufa determinou-se o conteúdo total de $\mathrm{P}$ por digestão nitro-perclórica e dosagem colorimétrica pelo molibdato de amônio (BATAGLiA et al., 1983), calculando-se, a partir desse dado, a razão de eficiência radicular (conteúdo total de P na planta/ área radicular).

Os dados obtidos foram analisados pelo programa MSTAT-C, sendo a comparação das médias feita pelo teste de Tukey a $5 \%$. Os dados referentes ao número de folhas e colonização micorrízica foram transformados para se ajustarem à distribuição normal, sendo o primeiro em raiz quadrada de $(x+0,5)$ e o segundo em arco-seno da raiz quadrada da porcentagem. Em relação ao substrato, embora seja um fator quantitativo, optouse, para sua avaliação, pela análise da variância com teste de médias.

\section{RESULTADOS E DISCUSSÃO}

Aos 65 dias de aclimatização, verificou-se que as mudas com G. clarum, no substrato sem matéria orgânica, apresentaram altura superior ao controle sem fungo (Quadro 3). Resposta similar foi observada, aos 93 dias, no substrato com $10 \%$ de matéria orgânica. Comparando-se os substrato, dentro do tratamento com FMA, verificou-se que as mudas com $10 \%$ de matéria orgânica mostravam altura superior àquelas sem matéria orgânica, desde a primeira avaliação, aos 32 dias. Contudo, somente aos 65 dias, as mudas do substrato com $20 \%$ de matéria orgânica apresentaram altura superior àquelas sem matéria orgânica. No tratamento sem FMA, observou-se, a partir dos 65 dias, maior altura nas mudas cultivadas com matéria orgânica (Quadro 3). Na avaliação do diâmetro à altura do colo, verificou-se, aos 93 dias, que as mudas com FMA foram superiores às sem FMA, nos substratos com 0 
Quadro 3. Altura, diâmetro à altura do colo e número de folhas ativas de bananeiras 'Nanicão', com e sem Glomus clarum, em substratos com três níveis de matéria orgânica, em três épocas de avaliação, sob condições de casa de vegetação

\begin{tabular}{|c|c|c|c|c|c|c|}
\hline \multirow{2}{*}{ Substrato } & \multicolumn{2}{|c|}{ Altura } & \multicolumn{2}{|c|}{ Diâmetro } & \multicolumn{2}{|c|}{$\mathrm{N}^{\circ}$ de folhas } \\
\hline & Com FMA & Sem FMA & Com FMA & Sem FMA & Com FMA & Sem FMA \\
\hline & \multicolumn{3}{|c|}{$\longrightarrow \mathrm{cm}$} & & \multicolumn{2}{|c|}{ folhas.pl ${ }^{-1}$} \\
\hline \multicolumn{7}{|c|}{32 dias } \\
\hline $0 \%$ & $2,27 \mathrm{Ba}$ & $2,60 \mathrm{Aa}$ & $0,55 \mathrm{Aa}$ & $0,61 \mathrm{Aa}$ & $3,94 \mathrm{Ba}$ & $4,31 \mathrm{Ba}$ \\
\hline $10 \%$ & $5,11 \mathrm{Aa}$ & $4,74 \mathrm{Aa}$ & $0,81 \mathrm{Aa}$ & 0,77 Аа & $5,38 \mathrm{Aa}$ & $5,38 \mathrm{Aa}$ \\
\hline $20 \%$ & 4,42 $\mathrm{ABa}$ & $4,93 \mathrm{Aa}$ & $0,76 \mathrm{Aa}$ & $0,82 \mathrm{Aa}$ & $5,75 \mathrm{Aa}$ & $6,31 \mathrm{Aa}$ \\
\hline \multicolumn{7}{|c|}{65 dias } \\
\hline $0 \%$ & $6,00 \mathrm{Ba}$ & $3,67 \mathrm{Bb}$ & $0,86 \mathrm{Ba}$ & $0,79 \mathrm{Ba}$ & $7,00 \mathrm{Ba}$ & $5,75 \mathrm{Bb}$ \\
\hline $10 \%$ & $18,10 \mathrm{Aa}$ & $16,13 \mathrm{Aa}$ & $2,21 \mathrm{Aa}$ & $2,00 \mathrm{Aa}$ & $9,75 \mathrm{Aa}$ & $9,56 \mathrm{Aa}$ \\
\hline $20 \%$ & $16,22 \mathrm{Aa}$ & $18,33 \mathrm{Aa}$ & $2,11 \mathrm{Aa}$ & $2,18 \mathrm{Aa}$ & $9,38 \mathrm{Aa}$ & $10,00 \mathrm{Aa}$ \\
\hline \multicolumn{7}{|c|}{93 dias } \\
\hline $0 \%$ & $11,78 \mathrm{Ba}$ & $4,68 \mathrm{Cb}$ & 1,37 Ca & $0,85 \mathrm{Cb}$ & $8,56 \mathrm{Ba}$ & $5,88 \mathrm{Bb}$ \\
\hline $10 \%$ & $28,77 \mathrm{Aa}$ & $23,03 \mathrm{Bb}$ & 3,19 Ba & $2,72 \mathrm{Bb}$ & $8,88 \mathrm{ABa}$ & 8,69 Aa \\
\hline $20 \%$ & $30,87 \mathrm{Aa}$ & $30,69 \mathrm{Aa}$ & 3,63 Aa & 3,59 Aa & $10,13 \mathrm{Aa}$ & $9,94 \mathrm{Aa}$ \\
\hline $\mathrm{CV}(\%)$ & 7,10 & & 5,39 & & 5,92 & \\
\hline
\end{tabular}

Médias seguidas por letras diferentes, maiúsculas na coluna e minúsculas na linha, dentro do mesmo fator, diferem pelo teste de Tukey ao nível de $5 \%$.

e 10\% de matéria orgânica (Quadro 3). Entretanto, considerando-se os substratos, notou-se, a partir dos 65 dias, maior diâmetro nas mudas com matéria orgânica, independentemente da presença ou ausência do FMA. No número de folhas ativas observou-se resposta idêntica, desde a primeira avaliação, aos 32 dias. Aos 93 dias, contudo, no tratamento com FMA, não se verificaram diferenças entre os substratos com $0 \%$ e $10 \%$ de matéria orgânica. Os dados mostraram, ainda, interação entre os substratos e os tratamentos com e sem FMA, verificando-se, a partir dos 65 dias, maior número de folhas nas mudas infectadas com FMA, cultivadas no substrato sem matéria orgânica (Quadro 3).

Pode-se observar que aos 65 dias, essas três variáveis apresentaram comportamento similar, nos três substratos empregados. Esse fato pode refletir um estádio de maior desenvolvimento das mudas que possibilitaria, assim, maior colonização das raízes pelo FMA, estando essas condições associadas a uma adaptação às condições edafoclimáticas experimentais e à instalação de uma simbiose eficiente. Cabe assinalar aumento considerável da colonização micorrízica, bem como a presença de diferenças significativas entre os tratamentos, com e sem FMA, a partir dessa época.

Diferentes estudos (SouZA et al., 1987; Sousa, 1994; VERMA e ARYA, 1998) têm mostrado a importância do esterco na composição de substratos para a produção de mudas. Entretanto, quando se emprega inóculo de fungo micorrízico deve-se avaliar, com mais atenção, a quantidade e o tipo de material empregado como adubo orgânico. SouzA et al. (1989) verificaram que a presença de esterco de curral no substrato proporcionou maior desenvolvimento das mudas de café, não sendo alterada a colonização micorrízica das raízes. De acordo com Almeida et al. (1988) a composição química do esterco é variável, devendo ser considerada na interpretação dos resultados experimentais.

Pela análise dos substratos pode-se observar que a adição de esterco induziu alterações nas propriedades químicas e físicas dos substratos, que podem justificar as respostas positivas encontradas nas variáveis de crescimento avaliadas. Deve-se considerar, contudo, que o emprego do G. clarum constituiu um fator adicional importante para maior desenvolvimento das mudas. Segundo MARSCHNER e Dell (1994), os fungos micorrízicos possibilitam um maior crescimento das plantas, basicamente, pelo aumento da absorção de nutrientes.

Na colheita, aos 95 dias, constataram-se diferenças na massa de folha e pseudocaule secos das mudas entre os três substratos, sendo os maiores valores obtidos com 20\% de matéria orgânica (Quadro 4). Quanto à massa de raízes secas, verificou-se que as mudas cultivadas com matéria orgânica mostraram valores 7,6 vezes superiores aos daquelas sem esse adubo. Considerando-se os tratamentos com e sem FMA, observou-se que as mudas infectadas foram 
Quadro 4. Massa de folha, pseudocaule e raízes secas, área e comprimento radicular específico de mudas de bananeira 'Nanicão', em substratos com três níveis de matéria orgânica, com e sem Glomus clarum, na colheita, aos 95 dias de aclimatização

\begin{tabular}{lccccc}
\hline Tratamentos & Folha & Pseudocaule & Raízes & $\begin{array}{c}\text { Área radicular } \\
\text { específica }\end{array}$ & $\begin{array}{c}\text { Comprimento } \\
\text { radicular específico }\end{array}$ \\
\hline & & & g.pl ${ }^{-1}$ & $\mathrm{~cm}^{2} \cdot \mathrm{g}^{-1}$ & $14 . \mathrm{g}^{-1}$ \\
$0 \%$ & $1,39 \mathrm{C}$ & $0,48 \mathrm{C}$ & $0,59 \mathrm{~B}$ & $140,46 \mathrm{~A}$ & $8,17 \mathrm{~A}$ \\
$10 \%$ & $8,92 \mathrm{~B}$ & $4,39 \mathrm{~B}$ & $4,33 \mathrm{~A}$ & $77,09 \mathrm{~B}$ & $3,59 \mathrm{~B}$ \\
$20 \%$ & $13,04 \mathrm{~A}$ & $6,52 \mathrm{~A}$ & $4,62 \mathrm{~A}$ & $88,24 \mathrm{~B}$ & $3,54 \mathrm{~B}$ \\
\hline Com FMA & $8,45 \mathrm{~A}$ & $4,13 \mathrm{~A}$ & $3,51 \mathrm{~A}$ & $100,77 \mathrm{~A}$ & $5,30 \mathrm{~A}$ \\
Sem FMA & $7,11 \mathrm{~B}$ & $3,46 \mathrm{~B}$ & $2,84 \mathrm{~B}$ & $103,09 \mathrm{~A}$ & $4,90 \mathrm{~A}$ \\
\hline CV $(\%)$ & 14,13 & 13,24 & 23,81 & 11,54 & 16,77 \\
\hline
\end{tabular}

Médias seguidas por letras diferentes na coluna diferem pelo teste Tukey ao nível de 5\%.

Quadro 5. Conteúdo total de P na planta, razão de eficiência radicular e porcentagem de colonização micorrízica de mudas de bananeira 'Nanicão', em substratos com três níveis de matéria orgânica, com e sem Glomus clarum, na colheita, aos 95 dias de aclimatização

\begin{tabular}{|c|c|c|c|c|c|c|}
\hline \multirow{2}{*}{ Substrato } & \multicolumn{2}{|c|}{ Fósforo } & \multicolumn{2}{|c|}{ Razão de eficiência radicular } & \multicolumn{2}{|c|}{ Colonização micorrízica } \\
\hline & Com FMA & Sem FMA & Com FMA & Sem FMA & Com FMA & Sem FM \\
\hline & \multicolumn{2}{|c|}{$\longrightarrow$ mg.pl ${ }^{-1}$} & \multicolumn{2}{|c|}{$\longrightarrow \mu \mathrm{g}$ P.cm ${ }^{-2} \longrightarrow$} & \multicolumn{2}{|c|}{$\%$} \\
\hline $0 \%$ & $4,96 \mathrm{Ca}$ & $0,59 \mathrm{Cb}$ & $39,10 \mathrm{Ba}$ & $21,52 \mathrm{Cb}$ & $61,24 \mathrm{Aa}$ & $0,00 \mathrm{Ab}$ \\
\hline $10 \%$ & $32,47 \mathrm{Ba}$ & 15,04 Bb, & $95,90 \mathrm{Aa}$ & $48,81 \mathrm{Bb}$ & $8,80 \mathrm{Ba}$ & $0,00 \mathrm{Ab}$ \\
\hline $20 \%$ & 45,15 Аа & $34,80 \mathrm{Ab}$ & 103,03 Аа & 95,93 Аа & $4,23 \mathrm{Ba}$ & $0,00 \mathrm{Ab}$ \\
\hline CV $(\%)$ & \multicolumn{2}{|c|}{11,02} & \multicolumn{2}{|c|}{14,30} & \multicolumn{2}{|c|}{34,42} \\
\hline
\end{tabular}

Médias seguidas por letras diferentes, maiúsculas na coluna e minúsculas na linha, dentro do mesmo fator, diferem pelo teste Tukey ao nível de 5\%.

superiores às não-infectadas, em massa de folha, pseudocaule e raízes secas, independentemente do substrato empregado (Quadro 4).

Weber e AMORIM (1994) verificaram resposta similar em mudas de mamoeiro infectadas com FMAs, as quais apresentaram, aos 120 dias após o transplantio, maior produção de matéria seca do que as não-infectadas. VERMA e ARYA (1998), trabalhando com matéria orgânica e fungos micorrízicos na aclimatização de mudas micropropagadas de bambu, observaram aumento significativo na altura e na biomassa seca das plantas.

A área e o comprimento radicular específico das mudas cultivadas em substrato sem matéria orgânica foram significativamente maiores do que com esse adubo. Por outro lado, essas variáveis não foram influenciadas pela presença e ausência de FMA (Quadro 4). A análise dessas características é importante para avaliar o potencial de crescimento de raízes finas visando à maior absorção de nutrientes. Estudos desenvolvidos por GAHOONIA e NIELSEN (1998) mostraram forte evidência da participação substancial das raízes finas na absorção de fósforo do solo.
A análise do conteúdo total de $\mathrm{P}$ das plantas revelou diferenças entre os três substratos, nos tratamentos com e sem FMA; o substrato com $20 \%$ de matéria orgânica apresentou os maiores valores. Observou-se, ainda, que o conteúdo de $\mathrm{P}$ foi maior nas mudas infectadas que nas não-infectadas, nos três substratos estudados (Quadro 5). Considerando-se a eficiência de absorção, no tratamento com FMA, as mudas dos substratos com matéria orgânica apresentaram uma razão de eficiência radicular maior que aquelas sem esse adubo. Por outro lado, no tratamento sem FMA verificou-se uma relação direta da razão de eficiência com os níveis de matéria orgânica, ocorrendo os maiores valores no tratamento com $20 \%$ de matéria orgânica. Comparando-se a presença ou a ausência de micorriza, observou-se que nos substratos com $0 \%$ e $10 \%$ de matéria orgânica, as mudas com FMA foram superiores às sem FMA, ao passo que com 20\% não houve diferença (Quadro 5).

MARSCHNER (1995) considerou a morfologia da raiz como o fator mais importante na eficiência de absorção de P. No entanto, CLARKSON (1985) assinalou que a absorção de $\mathrm{P}$ é regulada por mecanismos de 
feedback, que podem operar ao nível de planta como um todo ou ao nível celular. Pesquisas desenvolvidas por PEARSON e JAKOBSEN (1993) evidenciaram a influência do fungo micorrízico na absorção desse elemento, assim como a variação desse efeito entre as espécies fúngicas.

No tratamento com FMA, a colonização micorrízica, avaliada a partir da porcentagem de raízes colonizadas, mostrou diminuição acentuada no tratamento com adição de matéria orgânica, independentemente da quantidade adicionada (Quadro 5). Souza et al. (1991) não observaram alteração na colonização das raízes de mudas de cafeeiro, na presença de matéria orgânica no substrato (30\% em volume), enquanto VerMa e ARYA (1998) citaram aumento da colonização com o uso de matéria orgânica ( $25 \%$ em volume) em mudas de bambu.

\section{CONCLUSÕES}

1. A inoculação com G. clarum propiciou maior crescimento e maior número de folhas nas mudas de bananeira cultivadas no substrato sem matéria orgânica, a partir dos 65 dias de aclimatização.

2. As mudas infectadas com G. clarum apresentaram, nos três substratos estudados, conteúdo total de $\mathrm{P}$ superior às não-infectadas.

3. O substrato com matéria orgânica possibilitou maior desenvolvimento das mudas de bananeira, cv. Nanicão, independentemente da presença do fungo micorrízico G. clarum.

4. A adição de matéria orgânica ao substrato acarretou diminuição acentuada na colonização das raízes por G. clarum.

\section{REFERÊNCIAS BIBLIOGRÁFICAS}

ALMEIDA, D.L. de; SANTOS, G. de A.; DE-POLLI, H. et al. Manual de adubação para o Estado do Rio de Janeiro. Itaguaí:Universidade Rural, 1988. 179p. (Série Ciências Agrárias, 2).

BARROW, N.J. Phosphorus uptake and utilization by tree seedlings. Australian Journal of Botany, Bronx, v.25, n 6, p.571-584, 1977.

BATAGLIA, O.C.; FURLANI, A.M.C.; TEIXEIRA, J.P.F et al. Métodos de análise química de plantas. Campinas: Instituto Agronômico, 1983.46p. (Boletim Técnico, 78).

BOLAN, N.S. A critical review on the role of mycorrhizal fungi in the uptake of phosphorus by plants. Plant and Soil, Dordrecht, v.134, n.2, p.189-207, 1991.
CLARKSON, D.T. Factors affecting mineral nutrient acquisition by plants. Annual Review of Plant Physiology, Palo Alto, v.36, p.77-115, 1985.

CRONAUER, S.S.; KRIKORIAN, A.D. Rapid multiplication of bananas and plantains by in vitro shoot tip culture. HortScience, Alexandria, v.19, n.2, p.234-235,1984.

EMBRAPA. Serviço Nacional de Levantamento e Conservação de Solos. Manual de Métodos e Análise de Solo. Rio de Janeiro: EMBRAPA-SNLCS, 1979. n.p.

EMBRAPA. Serviço Nacional de Levantamento e Conservação de Solos. Manual de Métodos e Análise de Solo. Rio de Janeiro: EMBRAPA-SNLCS, 1997. 212p. (EMBRAPA-SNLCS. Documentos, 1).

FAO, Yearbook: Production. Rome: Food and Agriculture Organization of the United Nations, 1997. v.50.

GAHOONIA, T.S.; NIELSEN, E.N. Direct evidence on participation of root hairs in phosphorus $\left({ }^{32} \mathrm{P}\right)$ uptake from soil. Plant and Soil, Dordrecht, v.198, n.2, p.147152, 1998.

JORGE, L.A. de C. Recomendações práticas para aquisição de imagens digitais analisadas através do SIARCS. São Carlos: EMBRAPA-CNPDIA, 1996. 44 p.(EMBRAPACNPDIA. Circular Técnica, 1).

KOSKE, R.E.; GEMMA, J.N. A modified procedure for staining roots to detect VA mycorrhizas. Mycological Research, Cambridge, v.92, n.4, p.408-505, 1989.

LOVATO, P.E.; HAMMATT, N.H.; GIANINAZZIPEARSON, V.; GIANINAZZI, S. Mycorrhization of micropropagated mature wild cherry (Prunus avium, L.) and common ash (Fraxinus excelsior L.). Agriculture Science of Finland, Finland, v.3, n.3, p.297-302, 1994.

MARSCHNER, H. Beneficial Minieral Elements. In: MARSCHNER, H. (Ed.). Mineral nutrition of higher plants. London: Academic Press, 1995. p.405-416.

MARSCHNER, H.; DELL, B. Nutrient uptake in mycorhizal symbiosis. Plant and Soil, Dordrecht, v.159, n.1, p.89-102, 1994.

NIEMI, M.; VESTBERG, M. Inoculation of commercially grown strawberry with VA micorrhizal fungi. Plant and Soil, Dordrecht, v.144, n.1, p.133-142, 1992.

PEARSON, J.N.; JAKOBSEN, I. Symbiotic exchange of carbon and phosphorus between cucumber and three arbuscular mycorrhizal fungi. The New Phytologist, London, v.124, n.3, p.481-488, 1993.

RAVELLI-NETO, A.; LIMA, E.; ANJOS, L.H.C.; PEREIRA, M.G. Roteiro de aulas práticas de morfologia e física do solo. Seropédica: Instituto de Agronomia/ Departamento de Solos, 1994. 56p. 
SOUZA, A. da S.; ZAIDAM, H.A.;SOUZA, F.V.D.; SILVA, K.M.da; PAZ, O.P. da. Protocolo de micropropagação de bananeira através de ápices caulinares. Cruz das Almas: EMBRAPA-CNPMF, 1994. 2p. (EMBRAPA-CNPMF. Banana em Foco, 31).

SOUZA, C.A.S.; CARVALHO, M.M. de; SOUZA, P. de et al. Influência de micorrizas vesicular-arbusculares no crescimento de mudas de cafeeiro (Coffea arabica L.) em substrato com e sem matéria orgânica e diferentes doses de superfosfato simples. Ciência $e$ Prática, Lavras, v.11, n.2, p.177-189, 1987.

SOUZA, C.A.S.; OLIVEIRA, E. de; CARVALHO, M.M. de. Desenvolvimento de mudas de cafeeiro (Coffea arabica L. cv. Catuaí) micorrizadas nas condições de viveiro comercial, em substrato com e sem matéria orgânica e diferentes doses de superfosfato simples. Ciência e Prática, Lavras, v.13, n.3, p.269-278, 1989.

SOUZA, C.A.S.; SIQUEIRA, J.O.; OLIVEIRA, E. de; CARVALHO, J.G. de. Crescimento e nutrição de mudas de cafeeiro micorrizadas-efeito da matéria orgânica e superfosfato simples. Pesquisa Agropecuária Brasileira, Brasília, v.26, n.11/12, p.1989-2005, 1991.
SOUSA, H.U. de. Efeito de composições e doses de superfosfato simples no crescimento e nutrição de mudas de bananeira (Musa sp.) cv. Mysore obtidas por cultura de meristemas. 1994. 88f. Tese (Mestrado em Fitotecnia) - Escola Superior de Agricultura de Lavras.

SOUZA, J. da S.; TORRES-FILHO, P. Aspectos socieconômicos. In: ALVES, E.J. (Ed.). A cultura da banana: aspectos técnicos, socieconômicos e agroindustriais. Brasília: EMBRAPA-SPI/Cruz das Almas: EMBRAPA-CNPMF, 1997. p.507-524.

VERMA, R.K.; ARYA, I.D. Effect of arbuscular mycorrhizal fungal isolates and organic manure on growth and mycorrhization of micropropagated Dendrocalamus asper plantlets and on spore production in their rhizophere. Mycorrhiza, New York, v.8, n.2, p.113-116, 1998.

WEBER, O.B.; AMORIM, S.M.C. de. Adubação fosfática e inoculação de fungos micorrízicos vesiculoarbusculares em mamoeiro 'solo' . Revista Brasileira de Ciência do Solo, Campinas, v.18, n.2, p.187-191, 1994. 